\title{
artigo
}

Nascimento, E.P.L.; Vizelli, C.; Mariano, R.C.Z; Romano, T.C.R.;

Dimensionamento como dispositivo de fortalecimento e consolidação da Estratégia de Saúde da Família no município Campinas

\section{Ferramentas utilizadas no cuidado continuado em saúde mental no contexto da COVID-19: uma revisão integrativa}

Tools used in continuous mental health care in the context of COVID-19: an integrative review

Herramientas utilizadas en la atención mental continua en el contexto de COVID-19: una revisión integrativa

\begin{abstract}
RESUMO
A pandemia causada pelo SARS-CoV-2 trouxe desafios e mudanças que influenciaram significativamente o cuidado em saúde mental, onde manter a longitudinalidade, antes uma ferramenta potente de assistência, tem sido um desafio para as equipes de Saúde da Família. Objetivo: buscar na literatura quais os meios que podem ser utilizados na APS para minimizar o sofrimento mental em decorrência da pandemia. Método: a busca pela literatura a partir da Biblioteca Virtual de Saúde (BVS), pelo cruzamento dos descritores: "cuidado" and "covid-19" and "saúde mental", sendo encontrados 347 artigos. Resultados: Através dos critérios de inclusão e exclusão a amostra total foi composta por 4 trabalhos. Conclusão: Observou-se que o uso de tecnologias leves vem sendo empregado para minimizar os efeitos psicológicos provocados pela pandemia, seja a pacientes acometidos pelo vírus ou a população que procura meios alternativos para manutenção da saúde mental.
\end{abstract}

DESCRITORES: Cuidado; COVID-19; Saúde Mental.

\section{ABSTRACT}

The pandemic caused by SARS-CoV-2 brought challenges and changes that significantly influenced mental health care, where maintaining longitudinality, once a powerful assistance tool, has been a challenge for Family Health teams. This integrative review aims to search the literature for the means that can be used in PHC to minimize mental suffering due to the pandemic. The methodology adopted was the search for literature in the Virtual Health Library (VHL), by crossing the descriptors: "care" and "covid-19" and "mental health", with 347 articles being found. Through the inclusion and exclusion criteria the total sample was composed of 4 works. It was observed that the use of light technologies has been used to minimize the psychological effects caused by the pandemic, either to patients affected by the virus or the population that seeks alternative means to maintain mental health.

DESCRIPTORS: Care, COVID-19, Mental Health.

\section{RESUMEN}

La pandemia del SARS-CoV-2 trajo desafíos y cambios que influyeron significativamente en la atención de la salud mental, donde mantener la longitudinalidad, anteriormente una poderosa herramienta de asistencia, ha sido un desafío para los equipos de Salud de la Familia. Esta revisión integradora tiene como objetivo buscar en la literatura los medios que se pueden utilizar en la APS para minimizar el sufrimiento mental debido a la pandemia. La metodología adoptada fue la búsqueda de literatura en la Biblioteca Virtual en Salud (BVS), cruzando los descriptores: "atención" y "covid-19" y "salud mental", encontrándose 347 artículos. Mediante los criterios de inclusión y exclusión la muestra total estuvo compuesta por 4 trabajos. Se observó que el uso de tecnologías ligeras se ha utilizado para minimizar los efectos psicológicos provocados por la pandemia, ya sea en los pacientes afectados por el virus o en la población que busca medios alternativos para mantener la salud mental.

DESCRIPTORES: Cuidado, COVID-19, Salud Mental.

RECEBIDO EM: 11/11/2020 APROVADO EM: 01/12/2020

\section{Marlla Héllen do Nascimento Araújo}

Graduanda em Medicina pela Universidade Federal de Campina Grande.

ORCID: 0000-0002-4662-1924 


\section{Maria Zilda Melo Regis}

Graduanda em Medicina pela Universidade Federal de Campina Grande.

ORCID: 0000-0003-0933-4747

\section{Samara Amorim de Araújo}

Graduanda em Medicina pela Universidade Federal da Paraíba.

ORCID: 0000-0003-4839-4208

\section{Rômulo Kunrath Pinto Silva}

Graduando em Medicina pela Universidade Federal da Paraíba.

ORCID: 0000-0002-4110-2017

\section{Denise Mota Araripe Pereira Fernandes.}

Médica de Família e Comunidade da Secretaria Municipal de Saúde de João Pessoa e preceptora voluntária do Internato em Saúde Coletiva da Universidade Federal da Paraíba. Mestranda em Saúde da Família pela FACENE.

ORCID: 0000-0001-7804-882X

\section{Vilma Felipe Costa de Melo}

Doutora. Psicóloga. Docente do Mestrado em Saúde da Família pela FACENE. ORCID: 0000-0001-5721-3240

\section{INTRODUÇÃO}

A partir de dezembro de 2019 o mundo assistiu a Organização Mundial da Saúde (OMS) anunciar as primeiras notícias do aparecimento de uma nova linhagem da coronavírus, denominada de SARS-COV-2, vírus de rápida disseminação, que provoca a doença COVID-19. Os primeiros casos aconteceram na cidade de Wuhan, província de Hubei e se espalharam, em poucos meses, para o mundo, produzindo uma cronologia de transmissão que, em pouco tempo, justificou o alerta mundial de status pandêmico ${ }^{1}$.

A pandemia trouxe, para o Sistema Único de Saúde (SUS), desafios adicionais ao cuidado prestado. Desde março de 2020 a saúde mental das pessoas sofre novas ranhuras, tendo em vista a reorganização da sociedade e suas relações com o ambiente e o isolamento social necessário para minorar o derramamento do vírus. Diante da sua gravidade e das repercussões psicossociais que essa doença pode gerar, a atenção em saúde mental tem sido abordada como um dos desafios para o seu enfrentamento, pois o cuidado é direcionado, prioritariamente, às questóes clínicas e científicas para o desenvolvimento da cura e/ou da recuperação dos órgãos e sistemas atingidos ${ }^{2}$.

Os relatos dos pacientes atendidos pelas equipes de Saúde da Família sinalizaram mudanças dramáticas no sono, menores possibilidades para realização de atividade física segura, adesões a dietas mais empobrecidas em nutrientes e uso de substâncias aditivas com efeitos psicotrópicos que podem refletir na saúde mental ${ }^{3}$. Tal fato é preocupante, uma vez que estamos passando por mudanças que podem influenciar significativamente o cuidado em saúde mental pelo desmonte dos Centros de Atenção Psicossocial (CAPS) na atual gestão administrativa brasileira ${ }^{4}$. A morte, o adoecer e a doença, o isolamento social, as perdas de renda e trabalho, o acúmulo de dívidas e a incerteza em relação ao futuro são reconhecidos fatores com impactos na saúde mental. ${ }^{5}$

No âmbito da Atenção Primária à Saúde (APS) manter o cuidado longitudinal, antes uma potente ferramenta de trabalho e cuidado, tem adquirido matizes de desafios mais potentes, uma vez que se tornam difíceis a realização de visitas domiciliares e o retorno programado para continuidade do cuidado. A APS deve apresentar papel fundamental para a identificação de casos, acompanhamento e promoção da saúde mental, ações com as quais os Agentes Comunitários de Saúde (ACS) podem contribuir ${ }^{6}$. Dessa forma, as equipes de Saúde da Família vêm buscando o uso de recursos tecnológicos leves e criativos para manutenção do cuidar, além de servirem de monitoramento online, permitindo gerar dados em tempo real para informar os gestores ${ }^{7}$.

Nesse sentido, o presente trabalho objetiva buscar na literatura quais os meios e tecnologias leves que podem ser utilizados na APS para minimizar o sofrimento mental decorrente deste tempo pandêmico à população em geral. A partir dessa inquietação, somada à insuficiência de estudos que exponham ferramentas utilizadas no cuidado em saúde mental durante a pandemia, evidencia-se a necessidade de conhecer a temática e dominar o uso de tecnologias leves em desfavor do sofrimento mental.

\section{MÉTODOS}

O método adotado no estudo foi a Revisão Integrativa da Literatura, que permite a comparação de dados para estudos acerca da temática8, ou seja, consiste em uma maneira de sumarizar e reunir informações sobre as pesquisas avaliadas, possibilitando um compêndio científico do tema investigado? A revisão integrativa da literatura direciona a uma fonte de conhecimento atual sobre um problema para, assim, permitir avanços no setor saúde. No entanto, é dotada de um forte rigor metodológico, no qual as etapas exigidas devem ser claramente apresentadas ${ }^{8,10}$.

Foram seguidas as orientações do PRISMA (Preferred Reporting Items for Systematic Reviews and Meta-Analyses), 


\section{artigo}

Nascimento, E.P.L.; Vizelli, C.; Mariano, R.C.Z.; Romano, T.C.R.;

Dimensionamento como dispositivo de fortalecimento e consolidação da Estratégia de Saúde da Família no município Campinas

marcada por colocações que atestam que a revisão sistemática com ou sem metanálise é formada a partir de um questionamento clínico, sob uma metodologia estruturada e reprodutível por pares. ${ }^{11}$

Em seguida foi realizada a busca a partir da Biblioteca Virtual de Saúde (BVS), plataforma que possibilita busca simultânea nas principais bases de dados de literatura nacionais e internacionais. A investigação se deu por meio do cruzamento de Descritores em Ciências da Saúde (DECS), com conectivo booleano "and" conforme se segue: cuidado and covid-19 and saúde mental.

Os critérios de inclusão foram trabalhos publicados entre dezembro de 2019 e dezembro de 2020, texto completo, no Brasil e nos idiomas inglês e português, a maio de 2020 para as bases de dados. Já os critérios de exclusão foram trabalhos que não contemplassem os critérios de inclusão, duplicados, com acesso restrito ou que não fizessem referência, em seu título ou resumo, à temática central do trabalho. Os artigos participantes constam no Portal Literatura-Americana e do Caribe em Ciências da Saúde (LILACS), na biblioteca Scientific Electronic Library Online (SciELO) e no Medical Literature Analysis and Retrievel System Online (MEDLINE).

Após resultados obtidos na busca de dados, foram entrelaçadas as informações e elaborada uma tabela contendo a síntese dos achados sobre o tema.

Figura 1. Etapas de seleção dos artigos da pesquisa sobre o cuidado frente à pandemia da COVID-19.

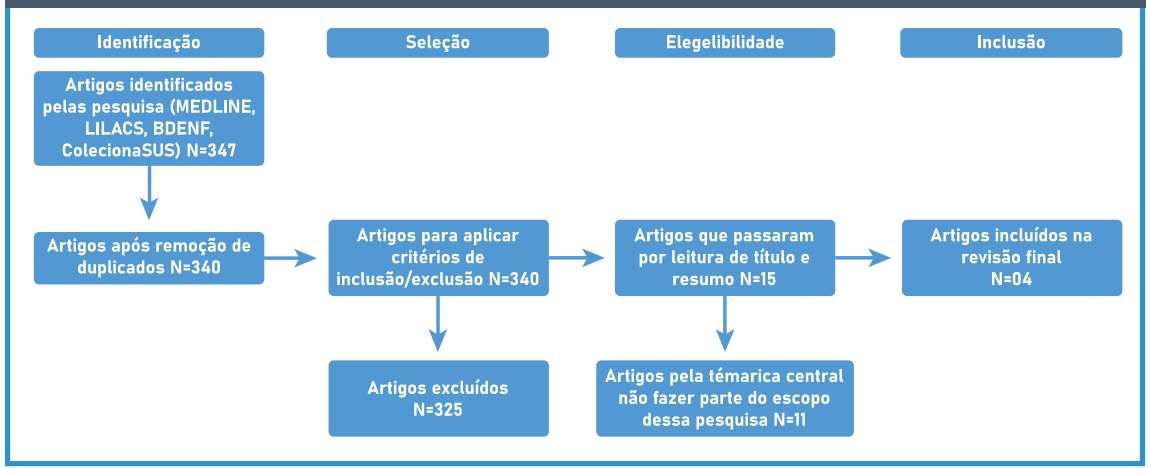

Fonte: própria dos autores (2020).

Figura 2- Síntese dos artigos incluídos na pesquisa.

\begin{tabular}{|c|c|c|c|c|}
\hline AUTORES & OBEJETO & $\begin{array}{l}\text { ABORDAGEM SOBRE A SAÚE } \\
\text { MENTAL }\end{array}$ & FERRAMENTAS UTILIZADAS & PLATAFORMA \\
\hline $\begin{array}{l}\text { Forlenza, OV, } \\
\text { Stella, F. } 2020\end{array}$ & $\begin{array}{l}\text { Avaliar o estado mental } \\
\text { de um grupo e idosos para } \\
\text { morbidade psiquiátrica do } \\
\text { SARS-COV-2 }\end{array}$ & $\begin{array}{l}\text { Há uma necessidade urgente de } \\
\text { gerar evidências cientificas sore } \\
\text { as necessidades psicológicas } \\
\text { e psiquiátricas dos idosos no } \\
\text { contexto pandêmico }\end{array}$ & $\begin{array}{l}\text { Os dados foram coletados pela } \\
\text { Escala Hospitalar de Ansiedade } \\
\text { e Depressão (HAD) e o Neurop- } \\
\text { sychiatric Inventory-Questionn } \\
\text { aire (NPI-Q) }\end{array}$ & MEDLINE \\
\hline $\begin{array}{l}\text { Monteiro-Junior, } \\
\text { RS et. al. } 2020\end{array}$ & $\begin{array}{l}\text { Fornecer uma visão geral } \\
\text { da resposta de três países } \\
\text { através de entrevistas } \\
\text { informais com profissionais } \\
\text { de saúde multidisciplinares }\end{array}$ & $\begin{array}{l}\text { Foi observado o uso de } \\
\text { tecnologias para aproximar } \\
\text { os idosos de seus familiares } \\
\text { e amigos e, assim, evitar que } \\
\text { se sintam solitários, evitando } \\
\text { o risco de contaminação o } \\
\text { potencial sofrimento mental }\end{array}$ & $\begin{array}{l}\text { Consultórios de rua, chamadas } \\
\text { telefônicas e de vídeo, aulas } \\
\text { online para prática de ecercício } \\
\text { físico. Ree de Solidariedade, } \\
\text { atividades de estimulação } \\
\text { social e cognitiva }\end{array}$ & MEDLINE \\
\hline $\begin{array}{l}\text { Castro-de-Araújo, } \\
\text { LFS., Machado, } \\
\text { DB. } 2020\end{array}$ & $\begin{array}{l}\text { Discutir os efeitos da } \\
\text { pademia do COVID-19 em } \\
\text { um país de baixa e média } \\
\text { renda, Brasil }\end{array}$ & $\begin{array}{l}\text { É recomendável que os cuidado- } \\
\text { res reduzam o número de pacien- } \\
\text { tes atendidos e que as familias } \\
\text { isolem-se junto com os idosos }\end{array}$ & $\begin{array}{l}\text { Acesso à psicoterapia é possível } \\
\text { de forma online, entretanto es- } \\
\text { sas práticas não estão completa- } \\
\text { mente regulamentadas. }\end{array}$ & SCIELO \\
\hline
\end{tabular}




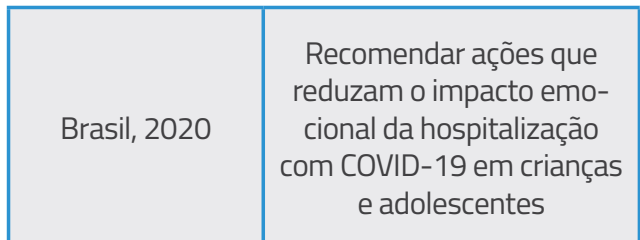

Fonte: Própria dos autores (2020).
A presença de uma rede socioafetiva é de extrema importância para minimizar os possíveis agravos na saúde mental de crianças durante a internação hospitalar na pandemia
Recursos tecnológicos (chamadas de video, áudios e mensagens de texto) permitem a comunicação das crianças e seus familiares, sendo uma forte rede de apoio para elas

\section{DISCUSSÃO}

As consequências da pandemia do novo coronavírus ultrapassaram as previsibilidades médicas e foram muito além das afetações sanitárias e orgânicas (biofisiopatológicas) da doença, ela afetou a saúde mental das pessoas nas suas integralidades: vivenciamos, também, "uma pandemia de sofrimentos mentais" em âmbito mundial. A pandemia da COVID-19 rompeu com o cotidiano das pessoas, deslocou-as de seus territórios existenciais, redirecionou costumes, separou as pessoas e rompeu (ainda que temporariamente) laços afetivos. Neste sentido, estudos afirmam que a pandemia da SARS-COV-2 tem enfraquecido determinantes do autocuidado em saúde mental ${ }^{12,13,14}$.

Por sua vez, o estudo de Forlenza e Stella, desenvolvido em um hospital terciário em São Paulo ao buscar avaliar o estado mental de um grupo de idosos para morbidade psiquiátrica durante a pandemia do novo coronavírus, observou que as queixas de prejuízo ao sono foram feitas por 57\% dos participantes, as quais incluem insônia, sono ineficiente ou sonolência durante o dia, além de sintomas de humor ${ }^{15}$. Tais aspectos são mais evidenciados em contextos de pandemia nessa faixa etária em razão dos próprios fatores biológicos relacionados à idade, comportamentos de saúde, fatores socioeconômicos e ambientais ${ }^{16}$.

Ainda sobre a saúde mental da população idosa em tempos de pandemia, um estudo traz que as taxas de suicídio entre idosos aumentaram durante este perío- do ${ }^{17}$, o que enfatiza a urgência de estudar o impacto do COVID-19 na saúde mental dessa população ${ }^{18,19}$. Nessa perspectiva, o estudo de Monteiro-Júnior e colaboradores observou a assistência a idosos na pandemia em 3 países: Brasil, Portugal e Noruega. O mesmo concluiu que, apesar dos países pertencerem a classificações distintas no Relatório de Desenvolvimento Humano, as estratégias utilizadas para mitigar o sofrimento mental foram semelhantes: Desenvolveram consultórios de rua, fizeram uso de chamadas telefônicas e de vídeo, forneceram aulas online para prática de exercício físico, montaram Redes de Solidariedade e estimularam a prática de atividades sociais e de carácter cognitivo na terceira idade ${ }^{20}$.

Castro-de-Araújo e Machado ressaltam que é difícil manter os cuidados com a população geriátrica durante a pandemia. Além disso, salientam as recomendações de que os cuidadores reduzam o número de pacientes atendido, assim cabe a família a maior parte do cuidado ${ }^{21}$. O uso de tecnologias interativas como videogames podem ser recursos a serem utilizados pelos idosos a fim de funcionar como uma alternativa a prática de atividade, ajudando a aliviar o comportamento sedentário ${ }^{22}$.

No âmbito da atenção terciária, o uso de tecnologias, como chamadas de vídeo, mensagens de textos e áudios, constituem ferramentas importantes para estreitar a distância entre os pacientes, contribuindo para melhora da sua saúde mental. Na população pediátrica, o Ministério da Saúde recomenda o incentivo da participação familiar como forma de acompanhar regularmente as crianças hospitalizadas na pandemia ${ }^{23}$. O uso dessas ferramentas não se restringe apenas à rede hospitalar, mas também à atenção primária.

No contexto da pandemia, alguns Agentes Comunitários de Saúde (ACS) monitoravam seus usuários por meio do WhatsApp e atendiam os grupos mais frágeis e vulneráveis que precisavam de atenção especial ${ }^{24}$, mesmo não havendo regulamentação pelas associações brasileiras ${ }^{23}$. Tais recursos se tornam valiosos nesse cenário ao diminuir a sensação de solidão nos usuários, por exemplo.

\section{CONCLUSÃO}

A continuidade do cuidado em saúde dos usuários da atenção primária à saúde tem sido prejudicada pela quarentena. Associado aos problemas de saúde já pré-existentes a população acaba desenvolvendo quadros de ansiedade e depressão que interferem na qualidade de vida. Compreender as necessidades da população neste momento é essencial, fazendo o uso de alternativas que aproximem a equipe de saúde aos usuários, como o uso de tecnologias de monitoramento de saúde online, WhatsApp, chamadas de vídeo e chamada telefônica.

Os estudos encontrados e discutidos nesta revisão possibilitaram ter uma ideia da dimensão da problemática, porém denota-se que poucos são os estudos que abordam o cuidado em saúde mental na atenção primária à saúde, fazendo-se que mais estudos na área sejam realizados para permitir encontrar soluçôes mais consistentes para o problema. -

\section{REFERÊNCIAS}

1. ORGANIZAÇÃO MUNDIAL DE SAÚDE. COVID-19: cronología de la actuación de la OMS. 2020 [acesso em 12 dez 2020]. Dis- ponivel em: https://www.who.int/es/news-room/detail/27-042020-who-timeline---covid-19 


\section{artigo}

Nascimento, E.P.L.; Vizelli, C.; Mariano, R.C.Z.; Romano, T.C.R.;

Dimensionamento como dispositivo de fortalecimento e consolidação da Estratégia de Saúde da Família no município Campinas

\section{REFERÊNCIAS}

2. Pavani FM, Silva AB, Olschowsky A, Wetzel C, Nunes CK, Souza LB. Covid-19 e as repercussões na saúde mental: estudo de revisão narrativa de literatura. Rev Gaúcha Enferm. 2021;42(esp):e 20200188. doi: https://doi.org/10.1590/19831447.2021.20200188

3. Nabuco G, Oliveira MHPP, Afonso MPD. 0 impacto da pandemia pela COVID-19 na saúde mental: qual é o papel da Atenção Primária à Saúde? Rev Bras Med Fam Comunidade. 2020 [15 dez 2020] 15(42):2532. DOI: 10.5712/rbmfc15(42)253

4. Cruz NFO, Gonçalves RW, Delgado PGG. Retrocesso da Reforma Psiquiátrica: o desmonte da política nacional de saúde mental brasileira de 2016 a 2019. Trabalho, Educação e Saúde. 2020 [15 dez 2020] 18 (3):e00285117. DOI: 10.1590/1981-7746-sol0028

5. Kabad, JF et al. A experiência do trabalho voluntário e colaborativo em saúde mental e atenção psicossocial na COVID-19. Cadernos de Saúde Pública. 2020 [21 dez 2020] 36 (9): e00132120. DOI: 10.1590/0102-311x00132120

6. Alcântara KD, Carvalho FS, Belo ML, Souto MG, Silva AME, Silva GA. Contribuições de Agentes Comunitários de Saúde para a construção do perfil de usuários da Atenção Básica com necessidades de saúde mental. Cad Saúde Colet, 2020;28(4):599-608. https:// doi.org/10.1590/1414-462X202028040014

7. Balanzá-Martínez $V$, et al. Lifestyle behaviours during the COVID-19 - time to connect. Acta Psychiatr Scand. 2020 May;141(5):399-400. DOI:0.1111/acps.13177. PMID: 32324252; PMCID: PMC7264786.

8. Mendes KDS, Silveira RCDCP, Galvão CM. Revisão integrativa: método de pesquisa para a incorporação de evidências na saúde e na enfermagem. Texto \& contexto enfermagem, 2008 [12 dez 2020] 17(4), 758-764. DOl: 10.1590/S010407072008000400018

9. Ursi ES. Prevenção de lesões de pele no perioperatório: revisão integrativa da literatura. Dissertação (Mestrado em Enfermagem). Ribeirão Preto: Universidade de São Paulo, Escola de Enfermagem de Ribeirão Preto); 2005.

10. Whittemore R, Knafl K. The integrative review: updated methodology. Journal of advanced nursing, 2005 [12 dez 2020] 52(5), 546-553. DOI:10.1111/j.1365-2648.2005.03621.x

11. Singh S. How to Conduct and Interpret Systematic Reviews and Meta-Analyses. Journal of the American College of Gastroenterology. 2017 [14 jun 2020]; 8, e93. Disponivel em: <www. nature.com/ctg>

12. Kallakuri S, Maulik, PK. Challenges facing individuals and researchers: suicide in India in the COVID-19 pandemic. The lancet Psychiatry, 2020 [12 dez 2020] 7(8): 49. DOI: 10.1016/S22150366(20)30254-6

13. Pruitt LD, Mcintosh LS, Reger G. Suicide Safety Planning During a Pandemic: The Implications of COVID 19 on Coping with a Crisis. Suicide and Life Threatening Behavior, 2020 [12 dez 2020] 50(3): 741-749. DOl:10.1111/sltb.12641

14. Ruiz MA, Gibson CM. Emotional impact of the COVID-19 pandemic on US health care workers: A gathering storm. Psycholog- ical Trauma: Theory, Research, Practice, and Policy, 2020 [12 dez 2020] 12 (S1): S153. DOI: 10.1037/tra0000851

15. Forlenza, OV, Stella, F. Impact of SARS-CoV-2 pandemic on mental health in the elderly: perspective from a psychogeriatric clinic at a tertiary hospital in São Paulo, Brazil. International psychogeriatrics, 2020 [12 dez 2020] 32 (10):1147-1151. DOI:10.1017/S1041610220001180

16. Lima-Costa, MF et al. ELSI-COVID-19 initiative: methodology of the telephone survey on coronavirus in the Brazilian Longitudinal Study of Aging. Cadernos de Saúde Pública, 2020 [22 dez 2020] 36 (Suppl 3): e00183120. DOI: 10.1590/0102-311X00183120.

17. Kluge , H H P. Declaração - Os idosos correm o risco mais alto com o COVID-19, mas todos devem agir para evitar a disseminação pela comunidade. Diretor Regional da OMS para a Europa. 2020 [13 dez 2020] Disponível em: http://www.euro.who.int/en/ about-us/regional-director/news/news/2020/04/supportingolder-people-during-the-covid-19-pandemic-is-everyonesbusiness

18. Courtin E, Knapp M. Isolamento social, solidão e saúde na velhice: uma revisão de escopo. Saúde e Assistência Social na Comunidade. 2017 [13 dez 2020]; 25 (3), 799 - 812. DOI: 10.1111/hsc. 12311

19. Fiorillo A, Gorwood P. As consequências da pandemia COVID-19 na saúde mental e implicações para a prática clínica. European Psychiatry. 2020 [13 dez 2020]; 63 (1), E32. DOI: 10.1192/j.eurpsy.2020.35

20. Monteiro-Junior, RS. et al. COVID-19 pandemic: a multinational report providing professional experiences in the management of mental health of elderly. International Psychogeriatrics, 32(10), 1153-1156. 2020. DOI: 10.1017/S1041610220001027

21. Castro-de-Araújo, LF SCa., Machado, DB. Impact of COVID-19 on mental health in a Low and Middle-Income Country. Ciência \& Saúde Coletiva, 25, 2457-2460. 2020. DOl:10.1590/141381232020256.1.10932020

22. BRASIL. Ministério da Saúde. Fundação Oswaldo Cruz. Centro de Estudos e Pesquisas em Emergências e Desastres em Saúde (CEPEDES/Fiocruz). Saúde Mental e Atenção Psicossocial na Pandemia COVID-19: Recomendações para o Cuidado de Crianças em Situação de Isolamento Hospitalar. [publicação on line]; 2020 [acesso em 22 dez 2020]. Disponível em: https://portaldeboaspraticas.iff.fiocruz.br/atencao-crianca/covid-19-recomendacoes-para-o-cuidado-de-criancas-em-situacao-de-isolamento-hospitalar/

23. Giovanella, L. et al. ¿Es la Atención Primaria de Salud Integral parte de la respuesta a la pandemia de Covid-19 en Latinoamérica? Trabalho, Educação e Saúde. 2020 [22 dez 2020]; 19: e00310142. DOI: 10.1590/1981-7746-sol00310

24. Corvos César Augusto, Peroni Bruno Bizzozero, Pintos-Toledo Enrique, Fernández-Gimánez Sofía, Brazo-Sayavera Javier. Beneficios de los videojuegos activos sobre parámetros de aptitud física relacionada con la salud: un comentario en tiempos de cuarentena. Rev. Méd. Urug. 2020 [22 dez 2020]; 36(4): 234248. DOI: 10.29193/rmu.36.4.11. 